\title{
EXPERT Powering electronic contact lenses: current achievements, challenges, and perspectives
}

Expert Rev. Ophthalmol. 9(4), 269-273 (2014)

\section{Zoltan Blum, Dmitry Pankratov and Sergey Shleev*}

Department of Biomedical Sciences, Faculty of Health and Society, Malmö University, SE-205 06 Malmö, Sweden *Author for correspondence:

Tel.: +46406 657414

Fax: +46406658100 sergey.shleev@mah.se

The recent media hoopla regarding 'smart', 'bionic', or more appropriately, electronically augmented contact lenses is analyzed in terms of real achievements coupled to the critically important issue of power management. Not depending on the availability, currently or in the near future, of to-the-purpose discrete or integrated electronic devices, power management, including delivery/supply and temporal sustainability, will be an outstanding issue if present-day technology should remain the only option. Radically different approaches have been taken to deliver electric power to electronically augmented contact lenses, that is, ranging from quite simplistic wire-based delivery assemblies, grossly inappropriate for end users, to various elaborate wireless designs drawing on over-the-air power delivery, as well as solar and electrochemical cells. Nonetheless, given the complex restrictions offered by a contact lens, conventional, even state-of-the-art, power management technology is at an impasse, and to ensure a bright future for smart lenses, radical technological measures need to be taken. Bridging the conceptual gap between fuel cells and supercapacitors, an ingenious novel approach to on-lens power management is presented: a charge-storing fuel cell, or alternatively, a self-charging capacitor, that is, a hybrid electric power device.

KEYWORDs: battery $\bullet$ electrical energy $\bullet$ electrochemical cell $\bullet$ fuel cell $\bullet$ hybrid electric power device - over-the-air electric power $\bullet$ smart contact lens $\bullet$ solar cell $\bullet$ supercapacitor

\section{Expert commentary}

Recently, specialized and popular media alike have been awash with reports on so-called smart contact lenses, including presentations from multinational corporations like Microsoft and Google [1-4]. Drawing on the advances within nanotechnology, it has become technically feasible to fit electronics, for example, sensors, transmitters, amplifiers, and even displays, within the confinements of a standard-sized contact lens [5-9]. As regards sensors, they can be designed to gauge various features of biomarkers present in lachrymal fluid, while the complete circuitry arrangement, form factor, and opacity can be chosen not to impair vision. Contact lenses are minimally invasive, and electronic lenses will offer real-time, non-traumatic biomarker probing, among many different possible applications. It should be emphasized, however, that whereas reports in popular media concerning smart contact lenses are stunningly impressive [1-4], actual, bona fide contact lens-based gadgets presented in the scientific literature are still quite far from real practical applications [5-11]. As the name implies, electronic circuitry relies on electric power, and even though the power demands of state-of-the-art micro- or even nano-electronics are minimal, some power is nonetheless needed. However, delivering electric power to contact lens-based gadgetry is by no means a trivial undertaking, especially since conventional technologies do not fit the bill (vide infra).

The actual power requirement of a comprehensive lens assembly is difficult to ascertain as it depends on the technology choices made for the discrete components. Research reports quote solid-state integrated circuits, or 'computers', which supposedly run on fractions of a microwatt, while, for example, radio transmitters in the $10-\mu \mathrm{W}$ range are available off-the-shelf. The state-of-the-art is certainly progressing and more advanced systems with even lower power consumption are being developed. As for the sensor as such, depending on the protocol used, the power issue is more or less complex, especially since the act 
of measurement may generate power rather than expending it. Hence, as regards sensors, power per se is less of an obstacle; rather it appears that other aspects, such as overall performance and robustness, as well as form factor/scale constitute the major challenges. To conclude, the power requirement of an electronic contact lens could vary from close to nothing, when a self-powered sensor only is incorporated into the device, up to milliwatts for light-emitting diode-based multi-pixel displays.

To a first approximation, one can simply divide all the possible approaches to deliver electric power to electronically augmented contact lenses into two groups, viz., wired and wireless. While there is not much to say about the first approach $[5,8,9]$, which is obviously a reasonable approach for scientific studies but certainly inappropriate for an end user, the second group is very diverse and includes over-the-air (OTA) electric power delivery [2,6,8,11], as well as solar [7] and electrochemical cells [12,13].

To the best of our knowledge, there is only one report in the literature concerning the design, construction, characterization, and also in vivo testing of contact lenses incorporating solar cells. This approach, using $500 \times 500 \times 10 \mu \mathrm{m}$ singlecrystal silicon solar cells, is definitely very interesting, at least for certain applications, if the performance of solar cells is further improved [7]. Still, quite an obvious disadvantage is related to the fact that electricity cannot be continuously provided, for example, when the eyes are closed. Indeed, incorporation of an additional storage device, that is, a capacitor, will be needed.

Using a somewhat simplistic approach, electrochemical cells can be divided into two categories, viz., batteries and fuel cells (FCs), that is, 'closed' and 'open' systems. Batteries, even contemporary devices like modern solid-state batteries [14], are simply not compatible with human contact lenses due to many technical, regulatory, and safety issues. Thus, to the best of our knowledge, there is not a single report concerning the design, construction, and characterization of contact lenses incorporating batteries. Among many challenges with battery usage in contact lens applications, one can mention a general technical problem, viz., volume limitations: the accessible surface area is approximately $1 \mathrm{~cm}^{2}$, while the thickness should not exceed $0.2 \mathrm{~mm}$ [15]. Moreover, the possible toxicity of conventional batteries, if leakage should occur, is also a very serious issue since the device is located directly on the human cornea. Furthermore, there are purely regulatory obstacles, at least in the EU [16]; according to the EU regulations, battery usage in disposable devices is prohibited. Lenses are unquestionably disposable and, hence, cannot be powered by batteries. Thus, given that on-lens conventional batteries are disqualified for any number of reasons and that wired power delivery is also inappropriate, OTA power delivery seems to be the only remaining option.

Consequently, the available pre-flight information on 'smart' or 'bionic' lenses refers to OTA power securement, based on radio frequency coupling in particular $[2,6,8,11]$. Other OTA protocols, not specifically involving lenses, draw on infrared and ultrasound. On the lens, energy is collected by means of a suitable antenna and intrinsically relayed to the active circuitry, that is, the receiver/transmitter and sensor assembly; the sensor output is fed into the transmitter and the signal transmitted is eventually decoded in an external receiver. An external radio frequency transmitter (doubling as a data receiver/decoder), to be in some sense worn by the subject, provides the energy needed to drive the lens ensemble. As initially stated, owing to spatial limitations (on the lens), and the issues concerning vision impediment and end user convenience, and to some extent cosmetics, the dimensions of the various components cannot be arbitrarily chosen. Also, the hitherto unresolved issue regarding possible detrimental effects of microwave radiation needs to be considered.

Given the challenges and fundamental difficulties facing developers of bionic lenses, perhaps best illustrated by the major innovator behind the concept tentatively stating that wholesale market availability is at least for 5 years in the future, OTA power delivery protocols are perhaps better relegated, or complemented rather, in favor of emerging power-on-the-lens technologies.

One of the remaining options is to generate the power necessary by converting in situ available energy into electric energy, that is, drawing on the mainstay of FC technology, or in other words, 'open' electrochemical cells. Indeed, as early as in 2011, a patent application, patent cooperation treaty, entitled 'flexible biofuel cell, device and method' was filed, with the priority date 24 March 2010, describing the possible usage of biological fuel cells (BFCs) to power bionic contact lenses [17]. Later, several papers about the performance of different BFCs in human lachrymal liquid were also published $[12,13]$.

BFCs are bioelectronic devices that convert chemical energy directly into electrical energy using, contrary to conventional FCs, biological catalysts, such as proteins, enzymes, organelles, and whole cells [18]. BFCs have been envisioned as electrical power sources for implantable bioelectronics [19], and many reports exist in the literature nowadays concerning implantable BFCs [20-23]. However, in vivo operation possesses some significant hurdles to overcome, since an implanted biodevice would require a long operational lifetime; in case of electronic contact lens applications, the stability issue is, however, moot. A general outline of a BFC incorporated in a contact lens is presented in Figure 1. Briefly, an FC consists of at least two electrodes, an anode and a cathode. On the anode, biofuel oxidation occurs and the electrons thus released are transferred, via an electric circuit, to the cathode, where the reduction (electron uptake) of the biooxidant ensues. In terms of fuel availability, several possible biofuels are present in the lachrymal fluid, for example, ascorbic acid, dopamine, and glucose, albeit in limited supply. On the other hand, molecular oxygen (the ubiquitous biooxidant), needed to accept the electrons extracted from the fuels, is abundantly available. Simplified redox transformations of three biofuels and a biooxidant occurring at the electrode surfaces are given below:

glucose $\rightarrow$ gluconolactone $+2 \mathrm{H}^{+}+2 \mathrm{e}^{-}$

ascorbate $\rightarrow$ dehydroascorbate $+2 \mathrm{H}^{+}+2 \mathrm{e}^{-}$

dopamine $\rightarrow$ dopamine $o$-quinone $+2 \mathrm{H}^{+}+2 \mathrm{e}^{-}$

oxygen $+4 \mathrm{H}^{+}+4 \mathrm{e}^{-} \rightarrow 2$ water 
The thermodynamic redox potentials of the gluconolactone/ glucose, dehydroascorbate/ascorbate, and dopamine o-quinone/ dopamine couples at $\mathrm{pH} 7.4$ versus the standard hydrogen electrode are approximately $-0.4,0.1$, and $0.2 \mathrm{~V}$, respectively, whereas the thermodynamic redox potential of the oxygen/ water couple is $c a .0 .8 \mathrm{~V}$. Thus, the maximal theoretical (open circuit) voltages of glucose/oxygen, ascorbate/oxygen, and dopamine/oxygen FCs are 1.2, 0.7, and $0.6 \mathrm{~V}$, respectively.

Glucose, being the target molecule par excellence as regards the electronic lens protocols that are actively pursued, is a low-abundance substance in lachrymal fluid, that is, about $50 \mu \mathrm{M}$ on average [24], even if values earlier reported are as high as $0.6 \mathrm{mM}$ [25]. For a glucose sensing application, it would be important to mention several recent reports in the literature showing that the tear glucose concentration is quite adequately correlated (that is, real-time amounts) to blood glucose levels $[5,26,27]$. Given that the overall dynamic volume of basal tears is in the 10- $\mu$ l range and that no known methodology can ensure that the entire volume is reliably and repeatedly exposed to the appropriate FC modules, glucose is an unlikely on-lens energy source, if conventional (bio) FCs are to be employed. As can be seen from Figure 2, the electric power obtained from glucose as a biofuel is in the microwatts range, viz., $0.02-22 \mu \mathrm{W}$. For an average situation, viz., basal tears with a glucose concentration close to $50 \mu \mathrm{M}$ [24], and a tear production rate of about $3 \mu \mathrm{l} / \mathrm{min}$ [28], the calculated electric power is only $c a .0 .3 \mu \mathrm{W}$. In fact, the fuel alternatives, even if significantly more abundant [25], that is, ascorbic acid, on average $0.1 \mathrm{mM}$ (reported values in the literature are from $23 \mu \mathrm{M}$ [29] up to $665 \mu \mathrm{M}$ [30]), and dopamine, on average $1 \mathrm{mM}$ (from $c a .0 .06 \mu \mathrm{M}$ [31] up to $c a .2000 \mu \mathrm{M}$ [32]), are almost equally inappropriate, if conventional technologies are to be relied upon. In spite of the higher concentrations, the extractable electric power would be in the microwatts range still (Figure 2) because of the relatively high thermodynamic redox potentials, and correspondingly, lower operating voltages of FCs (vide supra).

The calculations presented in Figure 2 have been done using a very simple equation, $p=n F U C v$, where $p$ is the electric power, $n$ the number of electrons released from one fuel molecule, $F$ the Faraday constant, $U$ the $\mathrm{FC}$ voltage, $C$ the fuel concentration, and $v$ is the tear production rate. Let us consider in detail all the parameters that have been taken into account in the calculations.

$U$ values are based on the maximal theoretical (open circuit) voltages of glucose/oxygen, ascorbate/oxygen, and dopamine/ oxygen FCs (1.2, 0.7, and 0.6 V, respectively). In reality, however, $U$ values and, correspondingly, $p$ values, will be much lower. Another factor is the tear production rate. It is well known that the tear production rate depends on many factors, such as the kind of lachrymal liquid produced by the eye, that is, basal, induced, and emotional tears, as well as the physiological state of the eye. Accordingly, the rate varies from less than $1 \mu \mathrm{l} / \mathrm{min}$ up to $23 \mu \mathrm{l} / \mathrm{min}$, and this broad range was taken into account in our calculations.

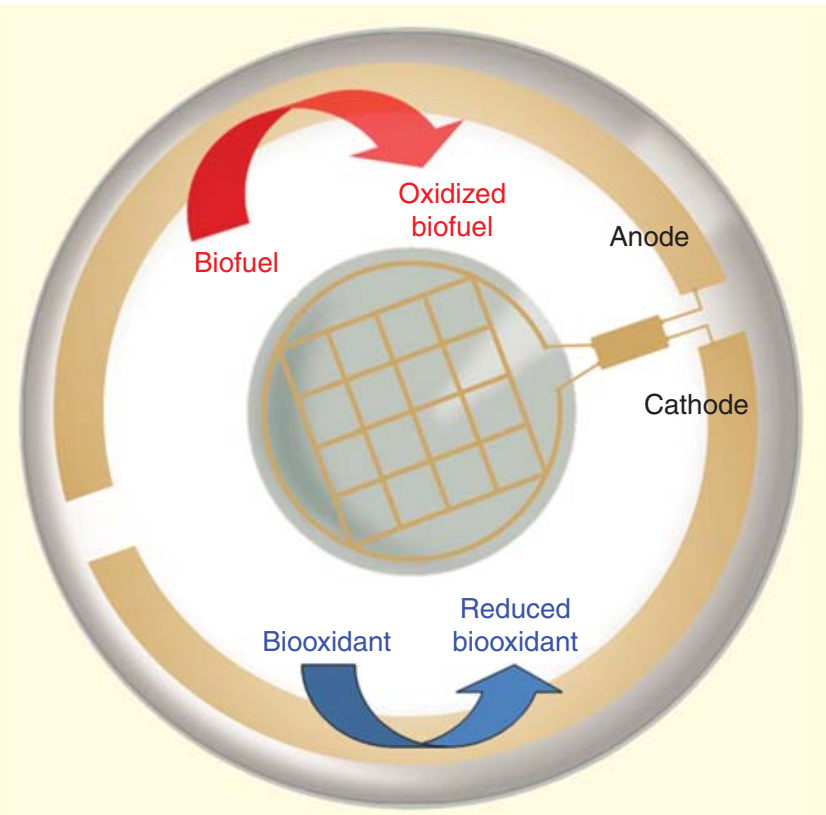

Figure 1. Contact lens with fuel cell fitted (the golden 'anode' and 'cathode' arcs at the lens perimeter) and model circuitry at the lens center.

Any on-lens assembly will be mitigated by the inherently static layout, and the mass transport (of fuels and their degradation products) will, by design, be unaided and, hence, impossible to influence. As a consequence, the power will be haphazardly delivered and the sensor (integrated or discrete) readings will be untrustworthy; relatedly, the information encoding and subsequent transmission will be marked with ambiguities linked to the intermittent supply of power. Clearly what is called for is a power source that is able to deliver a continuous supply of power throughout the sensing event, a supply that is consistently maintained when the information is encoded and transmitted. Taking into account both the spatial and physicochemical limitations, the suitability of OTA power delivery, or conventional (bio) FC technology, is highly questionable.

Recent developments regarding power source technology may very well present the solution; by a clever combination of an FC and a supercapacitor, low-yield, intermittent in situ energy conversion is utilized to charge a supercapacitor. Recognizing the problems with a discontinuous power supply, conventional capacitors were actually already inbuilt into electronic contact lenses with OTA power delivery [6,11]. In its present embodiment, the charge-storing $F C$, or alternatively, the self-charging capacitor, is built from high-end nanomaterials in a monocoque fashion, that is, the FC electrodes also serve as the capacitor conductors [33,34]. By design, hybrid capacitance, that is, double layer as well as electrochemical charging of the capacitor substructure, is relied on, thus enabling the storage of significant amounts of electric energy. The FC component draws on anodes and cathodes with immobilized catalysts. It should be 

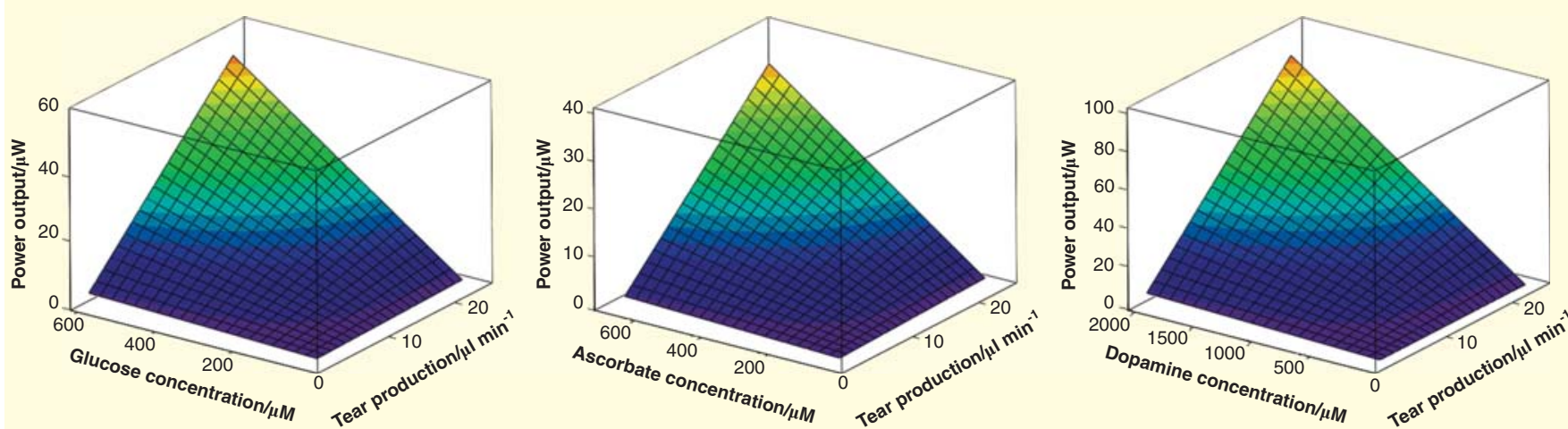

Figure 2. Calculated fuel cell power output as a function of reported biofuel concentrations and a broad physiological range of tear production rate.

emphasized that among many possible catalysts, enzymes working in direct electron transfer modes are the best, eliminating the need for mediators and making electrodeenzyme communication straightforward and less technically demanding. By running the charge-storing $F C$ in pulse mode, appropriately timing the cycle or pulse length, lowabundance fuels can be efficiently converted and the demands of consistent, temporally stable levels of power as regards the sensing, encoding, and transmission events can be reproducibly met.

To summarize, we believe that non-invasive sensing or evaluation of various biomarkers hold great promise for the future, especially if the information gathered can be effortlessly and accurately transferred to an external monitoring site. Focusing on non-invasiveness, and aiming at limited involvement of the individual, the combined efforts will most likely revolutionize the maintenance and treatment of any number of ailments, not only diabetes. To that end, the strict spatial confinement of a contact lens is singularly well suited as a test bed for the development of micro- and nano-sized implements, and the lessons learned will benefit all areas of real-time biomarker sensing.

\section{Expert commentary \& five-year view}

The researchers/technicians responsible at multinational corporations predict that market introduction of a glucosesensing lens is at least 5 years away. We believe that those predictions might be too optimistic, at least if they are not willing to abandon the power management technology they seem to be banking on. In our opinion, using the on-lens power management framework introduced here is intrinsically superior, in all aspects, to any OTA protocol or regime so far presented. In the hands of a visionary, technologically diverse, and financially robust company or consortium, we think that the time-to-market could be as low as 2 years. Nonetheless, in terms of maximizing corporate revenues, we firmly believe that targeting diabetes and glucose sensing to that end is less than optimal. However commendable the approach is, from a business point of view it would be more valuable to identify an at least equally populated but healthy and effluent target group. Thus, in our opinion, the first financially significant wave of non-invasive, passive remote sensing will most likely move through the fitness and wellness market. Those markets are well equipped and also prepared to accept and carry the costs for development, allowing the health sector to move in at lower price points at a secondary stage. As a concluding remark, we would like to emphasize that this review is based on the complete set of the information available, viz., publicly available (from popular media) and also specialized information with limited access, that is, from patents, scientific papers, and conference proceedings. However, it is obvious that the topic is very 'hot' and interesting for commercialization. It is well known that companies usually are reluctant to disclose crucial technical details, proprietary knowhow, and patentable ideas. Also, filed patent applications effectively hide the relevant particulars for a substantial time. Thus, in spite of the comprehensive analysis of the information available, our commentary presented above describing the state-of-the-art might be less than adequate, or even incorrect; also, the real 5 -year story could be completely different.

\section{Financial \& competing interests disclosure}

The authors have no relevant affiliations or financial involvement with any organization or entity with a financial interest in or financial conflict with the subject matter or materials discussed in the manuscript. This includes employment, consultancies, honoraria, stock ownership or options, expert testimony, grants or patents received or pending, or royalties. No writing assistance was utilized in the production of this manuscript.

No writing assistance was utilized in the production of this manuscript. 


\section{Key issues}

- Prototypes of smart electronic contact lenses are demonstrated.

- Conventional batteries cannot be used to power bionic lenses.

- Wireless (over-the-air) electric power supply is used nowadays in combination with incorporated conventional capacitors.

- Solar and fuel cells are reported as one of the possible approaches.

- Newly reported hybrid electric power sources (self-charging supercapacitors or charge-storing fuel cells) seem to be the best devices to power bionic lenses.

\section{References}

1. Ulanoff L. Google smart contact lenses move closer to reality. Available from: www.mashable.com/2014/04/21/googlesmart-contact-lenses-patents/ Mashabe 2014

2. CoolThings. Google smart contact lens lets diabetes patients monitor their glucose levels by the second. Available from:

www.coolthings.com/google-smart-contactlens/ CoolThings 2014

3. Barad J, Buckland D, Chang J, et al. Microsoft and University of Washington partner on electronic contact lens research. Available from: www.medgadget.com/2012/ $01 /$ microsoft-and-u-of-washington-partneron-electronic-contact-lens-research.html 2012

4. Orca S. Micro machines and opto-electronics on a contact lens. Available from: www.hplusmagazine.com/2009/11/20/ micro-machines-and-opto-electronicscontact-lense/ H Plus Magazine 2009

5. Chu MX, Miyajima K, Takahashi D, et al. Soft contact lens biosensor for in situ monitoring of tear glucose as non-invasive blood sugar assessment. Talanta 2011;83(3): 960-5

6. Lingley AR, Ali M, Liao Y, et al. A single-pixel wireless contact lens display. J Micromech Microeng 2011;21(12):125014

7. Lingley AR, Otis BP, Shen TT, et al. A contact lens with integrated micro solar cells. Microsyst Technol 2012;18(4):45

8. Yao H, Liao Y, Lingley AR, et al. A contact lens with integrated telecommunication circuit and sensors for wireless and continuous tear glucose monitoring. J Micromech Microeng 2012;22(7):075007

9. Yao H, Shum AJ, Cowan M, et al. A contact lens with embedded sensor for monitoring tear glucose level. Biosens Bioelectron 2011;26(7):3290-6

10. Thomas N, Laehdesmaeki I, Parviz BA. A contact lens with an integrated lactate sensor. Sens Actuators B Chem 2012; 162(1):128-34
11. Liao YT, Yao H, Lingley AR, et al. A 3-microW CMOS glucose sensor for wireless contact-lens tear glucose monitoring. IEEE J Solid-State Circuits 2012;47(1):335-44

12. Falk M, Andoralov V, Blum Z, et al. Biofuel cell as a power source for electronic contact lenses. Biosens Bioelectron 2012; 37(1):38-45

13. Falk M, Andoralov V, Silow M, et al. Miniature biofuel cell as a potential power source for glucose-sensing contact lenses. Anal Chem 2013;85(13):6342-8

14. Jones KS, Rudawski NG, Oladeji I, et al. The state of solid-state batteries. Am Ceram Soc Bull 2012;91(2):26-31

15. Phillips A, Speedwell L. Contact lenses. Elsevier; NY, USA: 2007

16. EU European Union Directive 2006 on Batteries and Accumulators. Available from: www.epa.gov 2006

17. Ruzgas T, Shleev S, Arnebrant T. Flexible biofuel cell, device and method. WO patent application. 2011.EP54554: 31

18. Yang J, Ghobadian S, Goodrich PJ, et al. Miniaturized biological and electrochemical fuel cells: challenges and applications. Phys Chem Chem Phys 2013;15(34):14147-61

19. Katz E. Implantable bioelectronics. Wiley-VCH; Weinheim, Germany: 2014

20. Cosnier S, Le Goff A, Holzinger M. Towards glucose biofuel cells implanted in human body for powering artificial organs: review. Electrochem Commun 2014;38: 19-23

21. Falk M, Narvaez Villarrubia CW, Babanova S, et al. Biofuel cells for biomedical applications: colonizing the animal kingdom. ChemPhysChem 2013; 14(10):2045-58

22. Katz E, MacVittie K. Implanted biofuel cells operating in vivo - methods, applications and perspectives - feature article. Energy Environ Sci 2013;6(10): 2791-803
23. Schroeder U. From in vitro to in vivo-biofuel cells are maturing. Angew Chem Int Ed 2012;51(30):7370-2

24. Taormina CR, Baca JT, Asher SA, et al. Analysis of tear glucose concentration with electrospray ionization mass spectrometry. J Am Soc Mass Spectrom 2007;18(2):332-6

25. Berman ER. Biochemistry of the eye. Plenum Press; NY and London: 1991

26. Baca JT, Finegold DN, Asher SA. Tear glucose analysis for the noninvasive detection and monitoring of diabetes mellitus. Ocul Surf 2007;5(4):280-93

27. Peng B, Lu J, Balijepalli AS, et al. Evaluation of enzyme-based tear glucose electrochemical sensors over a wide range of blood glucose concentrations. Biosens Bioelectron 2013;49:204-9

28. Eter N, Gobbels M. A new technique for tear film fluorophotometry. $\mathrm{Br} \mathrm{J}$ Ophthalmol 2002;86(6):616-19

29. Choy CK, Cho P, Chung WY, et al. Water-soluble antioxidants in human tears: effect of the collection method. Invest Ophthalmol Vis Sci 2001;42(13):3130-4

30. Gogia R, Richer SP, Rose RC. Tear fluid content of electrochemically active components including water soluble antioxidants. Curr Eye Res 1998;17(3): 257-63

31. Martin XD, Brennan MC. Dopamine and its metabolites in human tears. Eur J Ophthalmol 1993;3(2):83-8

32. Agarwal S, Agarwal A, Apple DJ, et al. Textbook of ophthalmology. Jaypee Brothers Medical Publishers; New Delhi: 2002

33. Pankratov D, Falkman P, Blum Z, et al. A hybrid electric power device for simultaneous generation and storage of electric energy. Energy Environ Sci 2014; 7(3):989-93

34. Pankratov D, Blum Z, Suyatin DB, et al. Self-charging electrochemical biocapacitor. ChemElectroChem 2014;2:343-6 